\title{
Sustainability of Tobacco Cessation Programs
}

The cost of tobacco cessation programs and outcomes data are concerns and impediments for providers and hospital systems interested in providing services that, if successful, can support a tobacco-free lifestyle. As revealed in this issue of RESPIRATORY CARE in the article by Sweeny et al, ${ }^{1}$ reliable outcomes and sustainable support are difficult. The authors presented a need to provide smokingcessation support to adolescent patients and their parents to quit using tobacco or to abstain during hospital admissions. ${ }^{1}$ Visible setbacks occurred for patients with asthma who were improving until a parent or caregiver entered the hospital room smelling like cigarette smoke. Their novel approach included a team of respiratory therapists serving as nicotine replacement therapy (NRT) specialists, who provided weekly counseling and free NRT products. This is a great start in supporting families who want a smoke-free lifestyle after discharge. Their objectives were to assist in-patients and caregivers first to abstain from tobacco use during hospital admissions, and second to quit using tobacco entirely. The authors reported that their program assisted families as they begin the cessation process and provided educational and behavioral tools to families after discharge to continue a smoke-free lifestyle. ${ }^{1}$ We applaud their efforts and the experience that they have shared with us.

The utilization of respiratory therapists as Certified Tobacco Treatment Specialists is an innovative approach to cessation implementation. As such, other hospitals may benefit from using respiratory therapists as tobacco treatment specialists to decrease overall admissions for asthma exacerbations as well as COPD exacerbations among adults. Additionally, respiratory therapists should be at the forefront of the discussion on tobacco cessation due to their expertise, frequent interactions with patients, and knowledge of lung disease. This is a critical and timely opportunity for respiratory therapists to engage in tobacco

The authors have disclosed no conflicts of interest.

Correspondence: Lynda T Goodfellow EdD RRT AE-C FAARC. E-mail: ltgoodfellow@gsu.edu.

DOI: $10.4187 /$ respcare. 07765 cessation counseling, particularly due to the evolving cigarette and tobacco market with the surge in electronic cigarettes (e-cigarettes) and vaping. However, respiratory therapists typically only engage with patients within the hospital setting, thus emphasizing a need to sustain longterm cessation programs.

\section{See the Related Article on Page 407}

So, what happens now? It is hard for practitioners to obtain follow-up patient data or to remain in touch after discharge because patients and their families are trying to get back into their normal routines. Adherence with asthma medication administration or physician office appointments is often suboptimal. ${ }^{2-4}$ Funding is generally not available to sustain efforts outside the healthcare facility. In the study by Sweeny et al, ${ }^{1}$ for example, grant funding covered the first 2 years of the program, at which time services were absorbed into the department's budget. This included the salary of the coordinators and other team members who provided counseling, the NRT products, and a Great American Smoke Out event at the facility (personal communication, January 20, 2020, Natalie Napolitano).

Longitudinal studies that follow patients and parents through follow-up clinics are needed so that supplies and products continue until cessation is completed, as well as obtaining health metrics of the children. Little to no funding opportunities are available, however, and therefore the cycle continues. Innovative and sustainable approaches to continuing tobacco cessation programs after discharge are urgently needed. Mobile health interventions may be beneficial for this population to engage and support tobacco cessation efforts after discharge. Additionally, these interventions may be sustainable long-term because they carry little to no cost for participants. The report by Sweeney et al ${ }^{1}$ presents an important beginning to tobacco cessation implementation in a pediatric setting, and we encourage future studies to examine the long-term feasibility, efficiency, effectiveness, and sustainability. 


\section{EDITORIAL}

\section{Lynda T Goodfellow}

Rachel E Culbreath

Georgia State University

Department of Respiratory Therapy

Atlanta, Georgia

\section{REFERENCES}

1. Sweeney L, Taylor L, Peurifoy J, Kauffman K, Napolitano N. Success of a tobacco cessation program for parents at a children's hospital. Respir Care 2020;65(4):407-412.
2. Cheng NG, Browne GJ, Lam LT, Yeoh R, Oomens M. Spacer compliance after discharge following a mild to moderate asthma attack. Arch Dis Child 2002;87(4):302-305.

3. Sin DD, Bell NR, Svenson LW, Man P. The impact of follow-up physician visits on emergency readmissions for patients with asthma and chronic obstructive pulmonary disease: a population-based study. Am J Med 2002;112(2):120-125.

4. Joo KR, Brewer TL, Matcham WA. Implementation of text message reminder system in the pediatric urgent care to improve compliance with asthma care follow-up. J Informatics Nurs 2019;4 (1):6-12 